\title{
Correction to: Optimal design of hydraulic fracturing in porous media using the phase field fracture model coupled with genetic algorithm
}

\author{
Sanghyun Lee ${ }^{1} \cdot$ Baehyun Min ${ }^{2} \cdot$ Mary F. Wheeler ${ }^{3}$
}

Published online: 10 September 2018

(C) Springer Nature Switzerland AG 2018

Correction to: Computational Geosciences (2018)

\section{2(3):833-849}

https://doi.org/10.1007/s10596-018-9728-6

The original print publication of this article unfortunately contains mistakes introduced during the production process.

There were serious flaws in expressing the variable vector " $x$ " throughout the article. Instead of “ $\curvearrowleft$ ”, it should have been expressed as " $\not$ ".

The Publisher apologizes for this error.

The online version of the original article can be found at https://doi.org/10.1007/s10596-018-9728-6.

\footnotetext{
Baehyun Min

bhmin01@ewha.ac.kr

Sanghyun Lee

lee@math.fsu.edu

1 Department of Mathematics, Florida State University, 208 Love Building 1017 Academic Way, Tallahassee, FL 32306-4510, USA
}

2 Department of Climate and Energy Systems Engineering, Division of Sustainable Systems Engineering, Ewha Womans University, Ewhayeodae-gil 52, Seodaemun-gu, Seoul 03760, Republic of Korea

3 Center for Subsurface Modeling, The Institute of Computational Engineering and Sciences, The University of Texas at Austin, 201 East 24th Street, Austin, TX 78712, USA 